\title{
H5N1 Avian Influenza Virus: An Overview
}

\author{
José Luiz Proença-Módena, Izolete Santos Macedo and Eurico Arruda \\ Ribeirão Preto Medical School (FMRP); University of São Paulo (USP)
}

\begin{abstract}
Avian influenza virus (H5N1) emerged in Hong Kong in 1997, causing severe human disease. In recent years, several outbreaks have been reported in different parts of Asia, Europe and Africa, raising concerns of dissemination of a new and highly lethal influenza pandemic. Although H5N1 has not been capable of sustaining human-tohuman transmission, the ability of the virus to undergo variation due to mutations and reassortment, clearly poses the possibility of viral adaptation to the human species. For this reason the World Health Organization has established that we are now in a phase of pandemic alert. Preparing for an influenza pandemic involves a great deal of awareness necessary to stop initial outbreaks, through the use of case recognition, sensitive and rapid diagnostic methods, appropriate therapeutic and preventive measures to reduce spread. Influenza pandemic preparedness involves coordinated pharmacologic and vaccinal strategies, as well as containment measures such as travel restrictions and quarantine approaches.
\end{abstract}

Key-Words: H5N1 avian influenza virus, pandemic influenza, oseltamivir.

The emergence of epidemics of acute respiratory highly contagious illness, known as influenza, has afflicted humans since remote times. The word influenza (from the latin 'influentia'), means "epidemic" and conveys original vague concepts that such epidemics resulted from combinations of occult 'influences' [1]. Annual outbreaks of influenza occur regularly in temperate regions of the world with remarkable seasonality, defined by peak incidence in the colder months of the year. Such annual outbreaks vary in severity, but usually cause high morbidity and mortality, mainly among infants, the elderly, and immunocompromised patients [1].

In recent years, notably since 1997, the increased circulation of highly pathogenic avian influenza has been consistently detected in poultry and wild birds in several countries, mostly in Asia. In addition, 271 human cases of avian influenza (59.1\% of them lethal) have been officially reported by the World Health Organization (WHO) as of this writing [2]. The occurrence of human infection outbreaks of avian influenza, yet relatively restricted, raises concerns of dissemination of a new and highly lethal influenza pandemic. However, the actual risk of such a pandemic remains undetermined. This paper presents an overview of avian H5N1 influenza virus biology and highlights currently available information about prevention and control.

\section{Biology of Influenza A Virus}

Influenza viruses are RNA viruses in the family Orthomyxoviridae, which includes four genera: Influenza A, $\mathrm{B}$ and $\mathrm{C}$ viruses and Thogotovirus, as recently proposed by the International Committee on Taxonomy of Viruses (ICTV)

Received on 5 July 2006; revised 4 December 2006.

Address for correspondence: Dr. José Luiz Proença-Módena. Departamento de Biologia Celular, Molecular e Bioagentes Patogênicos, Faculdade de Medicina de Ribeirão Preto - USP. Ribeirão Preto, São Paulo, Brazil. Zip code: 14040-900. Fax: (55-16) 3633-1786. Phone: (55-16) 3602-3055. E-mail: jlmodena@rbp.fmrp.usp.br.

The Brazilian Journal of Infectious Diseases 2007;11(1):125-133. (C) 2007 by The Brazilian Journal of Infectious Diseases and Contexto Publishing. All rights reserved.
$[3,4]$. They are enveloped negative-stranded RNA viruses that can be distinguished on the basis of antigenic differences in nucleocapsid $(\mathrm{N})$ and matrix $(\mathrm{M})$ proteins [1].

Influenza A viruses are roughly spherical $(120 \mathrm{nM})$ with glycoprotein spikes on the surface and genome consisting of eight RNA fragments that encode 10 proteins. The haemagglutinin (HA), neuraminidase (NA) and matrix (M2) proteins are embedded in the envelope lipid bilayer derived from the host cell [1] (Figure 1). The M1 protein underlying the envelope is the major determinant of virion morphology [5]. The nucleoprotein (NP) associates with each RNA segment to form the ribonucleoprotein (RNP) complex, which also contains small amounts of the three polymerase subunits. The nonstructural proteins NS1 and NS2 are found only in infected cells [1].

The surface glycoproteins HA and NA are critical for the biology of influenza virus. HA is responsible for the virus attachment to the cell surface, binding to sialic acid residues in cell membrane glycoproteins, thus triggering viral fusion and entry [6]. The proteolytic cleavage of HA by serine proteases present in the infected tissue exposes hydrophobic fusion domains that mediate membrane fusion. This is an important molecular determinant of host range and tissue pathology $[7,8]$. Some HA types can be cleaved by different serine proteases, what enables the virus to spread more efficiently in vivo $[9,10]$. The RNA-dependent RNA polymerase and the NS1 proteins of influenza virus also are determinants of viral pathogenicity and host range [11,12].

NA cleaves terminal sialic acid from glycoconjugates present on respiratory mucins, cells, and progeny virions [1,3]. This action is of key importance for the release of the virus from infected cells and, consequently, for the spreading of infection throughout the respiratory tract.

The antigenic diversity of HA and NA provides for influenza A virus subtyping. Sixteen HA and nine NA subtypes are currently recognized, with amino acid sequences differing by $30 \%$ or more [13]. Six HA (H1, H2, H3, H5, H7 and H9) and three NA (N1, N2 and N7) subtypes have been identified in strains of influenza virus causing human infection, but only 
Figure 1. Schematic diagram of influenza A virus. Inserted in the lipid bilayer are the haemagglutinin (HA), neuraminidase (NA) and matrix (M2) proteins. The M1 protein is localized underlying the viral envelope. The nucleoprotein and three polymerase proteins are associated with each of the eight single-stranded RNA segments (1-PB2, 2-PB1, 3-PA, 4-HA, 5NP, 6-NA, 7-M, 8-NS) to form the ribonucleoprotein complex (RNP).

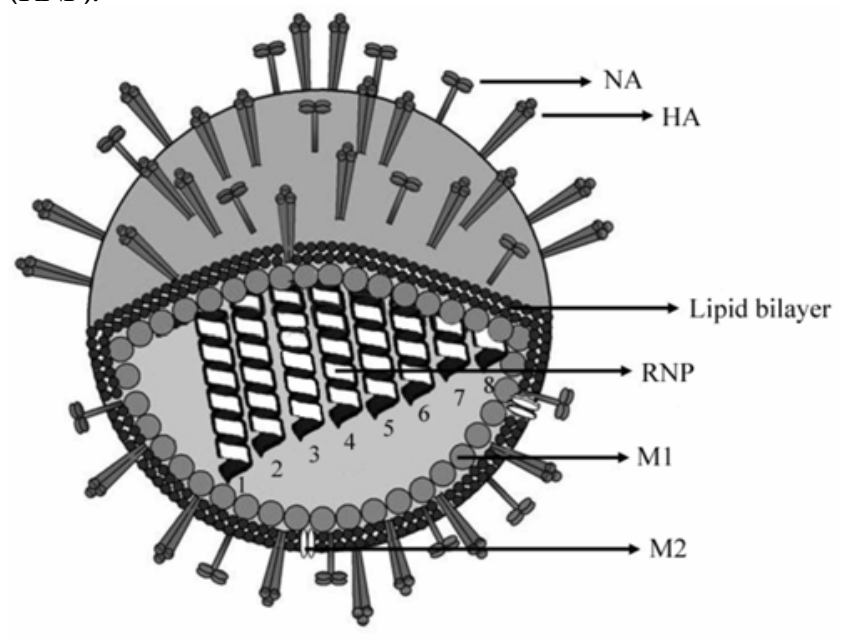

Figure 2. Schematic diagram of the replicative cycle of influenza A. Virus binds to sialic acid residues present on cell surface glycoproteins or glycolipids and internalization is mediated by endocytosis in clathrin coated vesicles. Endosomal acidification triggers the release of the genome in the cytoplasm host cell. Intact ribonucleoprotein complexes enter the nucleus, where transcription of viral RNA (vRNA) into messenger RNA (mRNA) and complementary RNA (cRNA) takes place, mediated by the viral RNA polymerase complex. The assembly of vRNAs into infectious viruses involves several cellular compartments and final budding occurs through the cell surface.

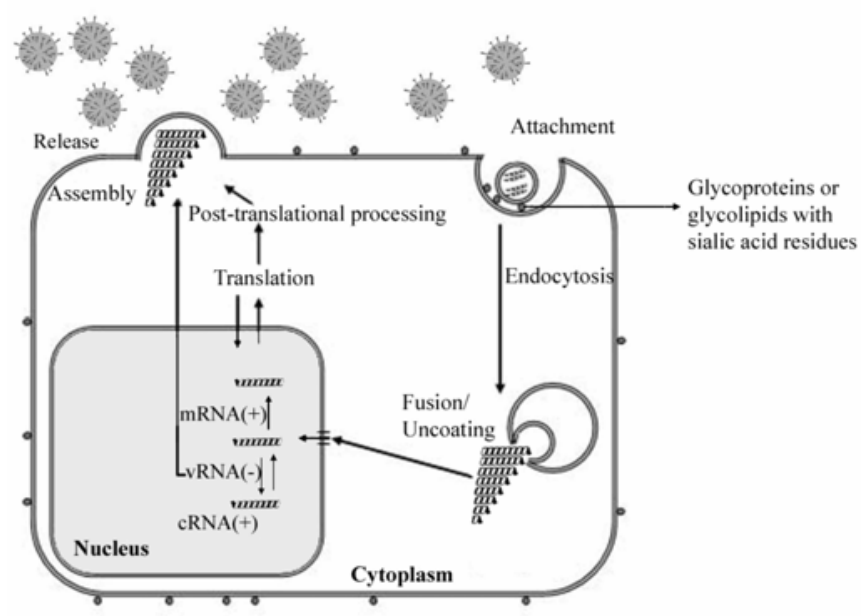

Figure 3. Schematic diagram of influenza A antigenic shift. These changes are caused by the acquisition of new gene segments thorough genetic reassortment between two virus strains infecting simultaneously the same host cell.

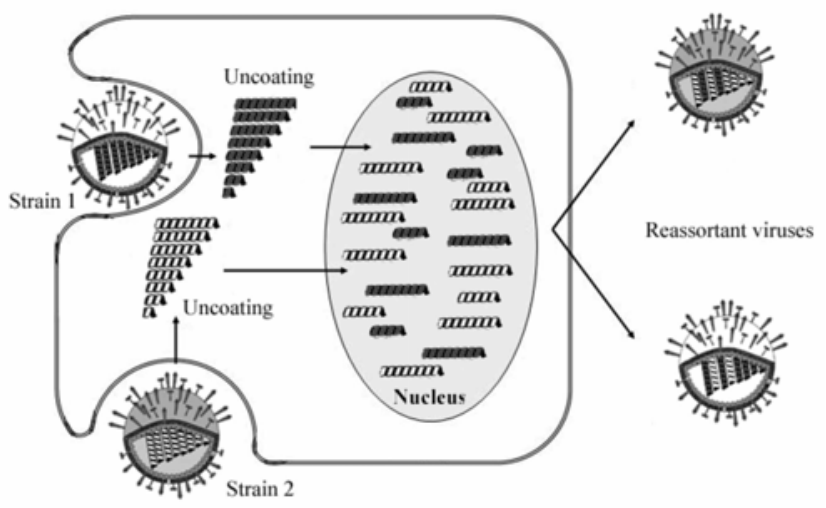

viruses of three HA (H1, H2 and H3) and two NA (N1 and N2) subtypes have remained in sustained circulation in the human population after causing pandemics [14].

After the binding of HA to sialic acid residues present in glycoproteins or glycolipids on the cell surface, influenza virus enters susceptible cells by receptor mediated endocytosis (Figure 2). This internalization mediated by clathrin coated vesicles is followed by the release of the genome into the host cell cytoplasm, a phenomenon dependent on endosomal acidification [1]. Transcription of the negative-stranded RNA into either messenger RNA (mRNA), which directs viral protein synthesis, or complementary RNA (cRNA), which serves as template for the viral RNA (vRNA) genome synthesis, is mediated by a viral RNA polymerase complex active in the nucleus of host cell [15]. Assembly and packaging of vRNAs into infectious virions involve several cellular compartments. Nuclear export is promoted by the binding of M1 viral protein to RNPs, followed by the assembly of nucleocapsids in association with the cytoplasmic membrane and budding through the cell surface. Only viruses with a full complement of genome segments are infectious [1].

During replication of influenza virus, antigenic changes can occur, resulting in the continued circulation of the agent in human populations. Minor changes, called antigenic drift, are caused by the accumulation of point mutations during transcription of the viral genes, owing to the lack of proofreading ability of the viral polymerase. Such changes, mainly those occurring in the genes coding for HA and NA, can potentially generate new strains of influenza virus that spread in annual seasonal outbreaks. Unchecked by the lack of significant pre-existing protective immunity in the population, these new strains can cause widespread symptomatic infections [16]. More significant changes, called antigenic shift (Figure 3), result from the acquisition of entirely new gene segments, through genetic reassortment between two virus strains simultaneously infecting the same host. This may result in the emergence of novel influenza virus subtypes that 
Table 1. Pandemics of influenza A and other important influenza events in the last one hundred years.

\begin{tabular}{cccll}
\hline Year of appearance & $\begin{array}{c}\text { Time of } \\
\text { circulation (yr) }\end{array}$ & Virus subtype & Common designation & Estimated deaths \\
\hline 1918 & 39 & H1N1 & Spanish & Asian \\
1957 & 11 & H2N2 & Hong Kong & 86,000 in the USA \\
1968 & Present & H3N2 & H4,000 in the USA \\
1997 & Present & H5N1 & Chicken or bird & 148 deaths from 2003 until October \\
& & & & 2006 \\
\hline
\end{tabular}

may have increased virulence, a fact that can be aggravated by the lack of prior significant protective immunity in the new hosts [17]. Important examples of emerging influenza subtypes generated by antigenic shift have caused catastrophic pandemics of influenza with great impact in human history [17]. Recently reported outbreaks of infection caused by an emerging influenza virus in Asia involved a few hundred human cases with high fatality rate [2]. Such outbreaks were caused by a highly pathogenic H5N1 reassortant avian influenza virus [18] and have caused great concern, generating worldwide reactions from health authorities and the media. These developments followed the crossing of the species barrier by an epizootic highly pathogenic influenza virus that spread alarmingly in aquatic and terrestrial birds in Southeast Asia [19].

\section{Epidemiology of H5N1 Avian Influenza Virus}

Influenza A viruses are distributed throughout the world, causing symptomatic and asymptomatic infections in many vertebrate species, including a large variety of birds such as ducks, geese, terns, shearwaters, gulls, chickens, quail, turkeys, pheasants, starlings and budgerigars, as well as in swine, horses, seals whales, gibbons, baboons, chimpanzees and humans [20-24]. Phylogentic studies of influenza A viruses reveal that aquatic birds are the source of all influenza viruses in other species [25].

Over the past 150 years at least four pandemics of influenza occurred at irregular intervals, including three in the $20^{\text {th }}$ century (Table 1). These have caused high attack rates in all susceptible age groups, with high morbidity and mortality. The most lethal influenza pandemic in modern history was the H1N1 Spanish flu, which killed approximately 100 million people around the world between 1918 and 1919. The origin of the 1918 pandemic remains an enigma, but it is now clear that the virus had features of an avian virus [26-28], and it appears that an intermediate host, such as swine was involved [29]. Swine are known to be susceptible to both avian and human viruses, and could have served as hosts for additional drift resulting in the accumulation of changes from the original avian strain [28,29]. The pandemics of “Asian flu” (H2N2) in 1957 and the "Hong Kong flu" (H3N2) in 1968 caused an estimated 1 to 3 million deaths [15].

A highly pathogenic H5N1 avian influenza virus was first isolated in 1996, from a goose in China [30] and in 1997 it caused death in poultry in Asia [30]. In general, avian influenza A viruses do not cause disease in humans and prior to 1997 only two cases of natural human infections by animal influenza virus had been documented [31,32]. However, since 1997, avian influenza virus H5N1 has caused many cases of influenza in humans [30]. In January 1998, Subbarao and collaborators [33] characterized an H5N1 virus isolated from a child who died from a fatal respiratory illness on May 21, 1997 in Hong Kong. It was shown that genes encoding all internal and nonstructural proteins were closely related to known genes of avian origin and that this virus retained a preference for binding to Sia2-3Gal-terminated sialylglycoconjugates receptors, a feature typical of avian influenza viruses [34]. It has also been demonstrated that the HA of H5N1 has basic amino acids adjacent to the cleavage site, allowing it to be cleaved by proteases other than trypsin-like ones [33,35]. This augmented susceptibility of HA to cleavage by proteases widely distributed in tissues and organs helps to explain the broad tissue tropism of this virus and, consequently, its enhanced pathogenicity. In fact, H5N1 virus in mammals is not restricted to the lungs, but extends to other organs, including the brain [35].

These H5N1 influenza viruses have continued to reassort and undergo antigenic drift, as well as extend their host range, what is reflected by the detections of infections in swine and feline [36,37], raising the possibility of a possible participation of these animals in the spread of avian influenza virus. In $2004,8(0.25 \%)$ of 3.175 vietnamese pigs tested were positive for H5N1 influenza viruses by serum neutralization assay and Western blot [37]. Pigs possess receptors (NeuAco2,3Gal and NeuAc $\alpha 2,6 \mathrm{Gal})$ with affinity for both influenza A viruses [38], which led to the hypothesis that swine may serve as a "mixing vessel" for the generation of reassortants [38].

Felines have also become new targets for H5N1 research. In Germany, in February 2006, 8 of 111 apparently healthy cats coming from Thailand tested positive for antibodies to H5N1 viruses [36]. This finding has raised the question whether cats can spread avian influenza virus. Rimmelzwaan and collaborators (2006) showed that experimentally infected cats shed virus in sputum and feces [39].

While other animals have been found to be susceptible to either natural or experimental infection by influenza avian influenza, aquatic birds, mainly ducks, are the most important reservoirs of the virus throughout the world [40]. In ducks, 
avian viruses replicate in the lung and in cells lining the intestinal tract, and are shed in high concentrations in feces [41]. Water contamination with duck feces is a logical mechanism for the transmission of avian influenza viruses among aquatic birds and from them to domestic avian and mammalian species. The majority of H5N1 genotypes are avirulent in ducks, indicating that these animals can ensure perpetuation of these viruses [14].

From 1997 through 2001, the HA of the various H5N1 genotypes remained antigenically homogeneous, but in 2002 marked antigenic drift was reported $[42,43]$ and a strain of the so called $\mathrm{Z}$ genotype emerged, which is highly pathogenic for ducks and other aquatic birds [19]. In February 2003, two members of a family in Hong Kong died of infection caused by an H5N1 influenza virus strain [44], antigenically and molecularly similar to the one that was highly pathogenic for ducks and chickens [42].

Human influenza virus is generally transmitted from person to person by large droplets, small-particle aerosols, and possibly by fomites, with hand contamination and subsequent selfinoculation [15]. Recent evidence of limited human-to-human transmission of H5N1 was reported during a large family cluster in Indonesia [45]. Epidemiologic and genetic sequencing data suggest that a 10-year-old boy contracted the virus from his aunt and then passed it on to his father. In total, six members of the family died of H5N1 infection [45].

Virtually all people who became infected with H5N1 influenza virus had been exposed to an infected bird. Plucking and preparing diseased birds; handling fighting cocks; playing with poultry, particularly asymptomatic infected ducks; and consumption of duck's blood or, possibly, undercooked poultry, have all been implicated [46, 47]. This relative lack of adaptation of avian H5N1 to be transmitted from person to person has so far prevented the establishment of an overt avian influenza pandemic.

\section{Prospects of an H5N1 Pandemic: Current Situation}

The World Health Organization has defined six stages of a global plan for influenza pandemic preparedness, including recommendations for control measures to be adopted before and during an overt pandemic period [48]. The so called interpandemic period comprises the first two stages, when no new influenza virus subtypes are detected in humans, but a circulating animal influenza virus subtype poses a substantial risk of inducing human disease. The period called pandemic alert includes three phases: phase three, the one we are at as of this writing, is defined by the presence of detectable human cases of influenza caused by a new influenza virus subtype, but with limited human-to-human spread. In phase four human-to-human transmissions occurs, but only in small clusters, whereas in phase five such transmission occurs in larger clusters. Phase six is defined by a sustained transmission of a new flu subtype worldwide. Hundreds of cases of avian influenza (H5N1) infection in humans have been reported $[49,50]$, including a considerable number of fatal cases (Figure
4). The fatality rate among hospitalized patients has been high and all age groups have been affected [2].

The incubation period of avian influenza (H5N1) has been from two to eight days, and in most cases the disease occurs within two to five days after exposure. Initial symptoms in most H5N1 infected patients were those of influenza-like illness with high fever (typically of more than $38^{\circ} \mathrm{C}$ ) and lower respiratory tract symptoms [46]. Some patients also reported diarrhea, vomiting, abdominal pain, pleuritic pain, and bleeding from the nose and gums in the early stages of illness [49,51]. Two patients with encephalopathy and diarrhea without apparent respiratory symptoms have been reported [52]. Lower respiratory symptoms are usually found at presentation and include, in most cases, respiratory distress, tachypnea and inspiratory crackles and sputum production, which is, sometimes, bloody. Almost all patients have clinically apparent pneumonia with radiographic changes including diffuse, multifocal or patchy infiltrates; interstitial infiltrates and segmental or lobular consolidation with air bronchograms. Progression to respiratory failure has been associated with bilateral, ground-glass, diffuse infiltrates and manifestations of the acute respiratory distress syndrome. Other complications have included pulmonary hemorrhage, pneumothorax, ventilator-associated pneumonia, pancytopenia, Reye's syndrome and sepsis syndrome without documented bacteremia [46].

The enhanced pathogenicity of the H5N1 virus seems to be multifactorial. The factor most frequently alluded to is the existence in the haemmaglutinin of a polybasic amino acid region that renders it susceptible to cleavage by proteases present in multiple tissues, whereas in the interpandemic influenza subtypes this cleavage is usually performed by proteases more restricted to the respiratory tract $[33,35,46]$. Recently, comparison of human, avian, and swine HA structures have shown that HA of a highly pathogenic H5N1 strain (Viet04) is closely related to that of 1918 human influenza [53]. In addition, a specific substitution in the polymerase basic protein 2 (Glu627Lys) enhances viral replication [54,55] and a substitution in nonstructural protein 1 (Asp92Glu) confers increased resistance to elements of the host's innate immune response $[19,56]$.

\section{Prevention and Control}

Three international organizations coordinate the global H5N1 influenza surveillance effort: The World Health Organization (WHO) monitors human cases, while the World Organization of Animal Health (OIE) and the Food and Agriculture Organization (FAO) collect information of H5N1 in birds and other animals. It is clear that a rapid detection method for H5N1 will play a critical role in the control of a rapidly spreading flu pandemic, especially with regard to the ability to define the beginning of a new pandemic, so that prophylactic and contention measures can be adopted.

An ideal test for the detection of pandemic H5N1 virus, in addition to being highly sensitive, should be straightforward, 
Figure 4. World map depicting distribution of confirmed human cases of H5N1 avian influenza and occurrence in poultry and wild birds from 2003 to October 2006.

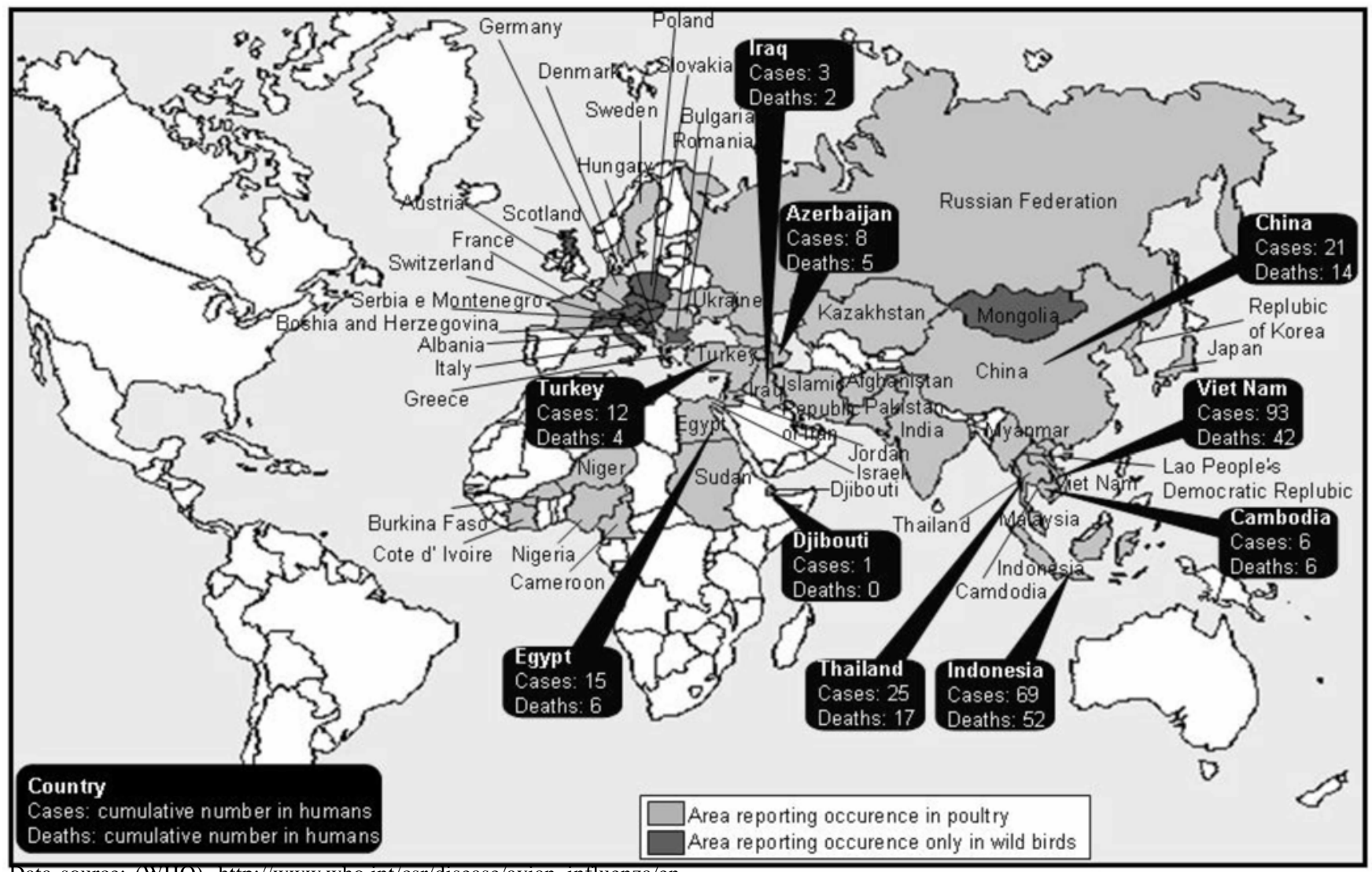

Data source: (WHO), http://www.who.int/csr/disease/avian_influenza/en.

quick to perform in a pandemic situation and capable to distinguish avian H5N1 from seasonal influenza. While there is a range of assays for the diagnosis of influenza $\mathrm{A}$, there is currently no ideal test displaying all such features. Influenza virus isolation in cell cultures such as Madin-Darby canine kidney cells (MDCK) followed by confirmation by immunofluorescence with $\mathrm{H} 5$ specific monoclonal antibodies has been attempted, but it may be laborious and limited to specialized laboratories by biosafety reasons [57]. Direct viral detection in clinical samples by immunoassay is also possible, but lacks sensitivity and specificity to reliably distinguish H5N1 strains from seasonal flu. Of interest, rapid antigen detection assays developed specifically for H5N1 are likely to miss drifting strains. NS1 protein, which exists in a specific form in avian influenza, could be used as a target for rapid diagnostic test that can potentially distinguish avian influenza from typical non-avian human influenza virus [58]. Serologic tests can be made very specific, but lengthy turnaround times hamper their use in the event of a pandemic.

Molecular diagnosis of H5N1 based on viral RNA detection by RT-PCR is potentially the most sensitive test and current real time methodology, as reported by $\mathrm{Ng}$ and collaborators (2005) [59], can shorten the turnaround time over the conventional RT-PCR assays in order to meet the needs of a pandemic situations [57,60].
Reducing the likelihood of interspecies transfer to combat the emergence of new influenza viruses in humans has been at the center of currently available methods for pandemic influenza control. In the case of H5N1, this has been attained through the culling of infected poultry, which reduces the viral pool available in the environment and reduces chances for its transmission to humans. This approach proved to be effective in several occasions, such as in 1997 in Hong Kong and in 2003 in The Netherlands, yet at the price of serious economic losses [61]. The use of an H5N1 vaccine in poultry can be an alternative solution. A recombinant adenovirus-based vaccine that protected chickens from a lethal intranasal challenge with VN/ 1203/04 H5N1 virus was recently proposed [62].

Any strategies to prevent avian influenza A (H5N1) in humans in a nonpandemic setting should be adopted as recommended by the WHO $[48,63]$. Patients with H5N1 should be treated in a combination of standard, contact, droplet, and airborne isolation precautions. They should be housed alone in a negative-pressure room or in a single room with closed door. If a single room is not available, patients should be housed in designated multibed rooms or wards. The beds should be at least $1 \mathrm{~m}$ apart from each other and preferably separated by a physical barrier. Health care workers should use high-efficiency masks (NIOSH-certified N-95 or equivalent), long-sleeved cuffed gowns, face shield or eye 
goggles, and gloves. The number of health care workers with direct contact with the patients, as well as visitors, should be limited. The health care workers or household contacts should monitor temperature twice a day and report any febrile event (temperature $>38^{\circ} \mathrm{C}$ ) and when this occur, they should undergo appropriate diagnostic testing for $\mathrm{H} 5 \mathrm{~N} 1$ and, if confirmed, should be treated immediately for 7 to 10 days. People that travel to a high risk area should avoid direct contact with chickens, ducks and geese, besides avoid touching feces or surfaces contaminated with secretions. Travelers should also consult a health care center if they become ill with respiratory symptoms and fever within 10 days after returning from an affected area [48, 63].

In a pandemic situation, contention and physical containment approaches could be adopted. The monitoring of travelers that arrive in a country, with quarantine approaches, the closure of agglomerating places, such as public transportation and schools, could be necessary actions $[48,63]$. However, the best strategy to combat a pandemic flu is a rapid and effective vaccine production. At the moment, the immunizations with formalin-inactivated or live-attenuated multivalent influenza virus's vaccine are methods available only for preventing interpandemic influenza.

Inactivated influenza virus vaccine is used prior to the influenza season. This vaccine is produced using one strain of influenza B and two strains of subtypes H3N2 and H1N1 of influenza A virus. This composition is determined annually on the basis of the antigenicity of influenza viruses monitored through the global World Health Organization surveillance network. The standard medium for influenza virus propagation before its inactivation and formulation for use in vaccines is fertilized chicken eggs. The viruses are replicated to high titers in allantoic fluid and purified and concentrated by zonal centrifugation or column chromatography and inactivated with formalin or $\alpha$-propialactone. This vaccine takes about 6 months to be produced, which is a long time to control a fast-moving flu pandemic [3]. In addition, the use of embryonated eggs is a limited strategy to produce vaccine against highly pathogenic flu virus, such as $\mathrm{H} 5 \mathrm{~N} 1$, because this virus can kill the embryos before high levels of virus are achieved, as observed with the H5N1 1997 Hong Kong virus [64].

A live-attenuated vaccine administered intranasally was licensed in the United States in 2003, where it has become an option for healthy persons aged 5 to 49 years [15]. This vaccine is composed by three different live flu viruses that were generated by cold attenuation. These viruses can replicate in the nose and throat (lower temperature), but not in the lower respiratory tract where temperature is higher $[15,65]$. This approach can become more practical for prevention of a possible avian influenza pandemic, since fertilized eggs are not used for virus propagation. However, cold adaptation of H5N1 influenza pandemic virus if possible, may take long, what might render this approach cumbersome. Therefore, a strategy by which attenuation can be achieved in a single step by transfer of attenuating genes should be advantageous to generate an effective vaccine in a pandemic situation. Reverse genetics is the best strategy currently available to attain this goal.

The strategies of reverse genetics (Figure 5) has been employed to rapidly produce candidate vaccines against pandemic flu viruses [66-68]. Briefly, this approach consist in the assortment of the HA and NA genes of the pandemic H5N1 influenza virus strain, previously cloned into plasmids, with the six remaining genes from A/Puerto Rico 8/34 (H1N1), a standard avirulent strain of influenza A virus, also cloned into plasmids. The eight plasmids are then transfected into mammalian cells, generating the desired vaccinal reassortant influenza virus strain [3]. Beforehand, the HA proteolytic cleavage site is removed to render the vaccinal virus avirulant while retaining its immunogenicity before cloning of the sixplasmid system. Recently, new systems have been developed reducing the number of plasmids required for the reverse genetics strategy, resulting in production of influenza A virus in Vero cells with high efficiency [69].

Other investigational approaches have been explored in H5N1 vaccine development, including immunization based in the utilization of adenovirus vector [62], DNA vaccine encoding a conserved nucleoprotein antigen [70], recombinant HA produced in insect cells [71], virosomes incorporating surface glycoproteins [72], and M2 protein conjugated with the hepatitis B virus core [73].

Antiviral drugs, especially neuraminidase inhibitors, could significantly help in the control of a future influenza pandemic caused by H5N1 or other strains by reducing viral loads in patients, reducing lower respiratory tract complications and hospitalizations, as well as decreasing potential person-toperson transmission. The use of these antiviral drugs can be particularly important to protect health care workers from H5N1 infection. The first antiviral drugs described against influenza were the adamantanes, amantadine and rimantadine. These compounds are M2 ion channel blockers that inhibit influenza A replication at the uncoating step [74]. However, the efficacy of such antiviral drugs is limited by the rapid emergence and transmission of drug-resistance variants [75]. Influenza virus resistance to amantadine-rimantadine emerges in approximately one third of treated patients [76] and is sometimes present naturally, including in recent human isolates of $\mathrm{H} 5 \mathrm{~N} 1$ virus [74].

Neuraminidase (NA) inhibitors, such as zanamivir and oseltamivir, were synthesized after the crystal structures of influenza NA complexes with sialic acid and the sialic acid derivative 2-deoxy-2,3-dehydro-N-acetyl-neuraminic acid were determined $[77,78]$. These inhibitors block the active site of the NA enzyme, inhibiting virus release from infected cells and spread within the respiratory tract [79]. This drug can potentially offer protection against any influenza virus that might emerge in humans, as the NA enzymatic active site seems to be highly conserved among all influenza viruses [80]. Studies have shown that either zanamivir or oseltamivir are effective against avian influenza virus (H5N1) [81,82]. Therefore, they hold great 
Figure 5. Schematic diagram of the reverse genetic strategy to produce candidate vaccines against pandemic flu viruses.

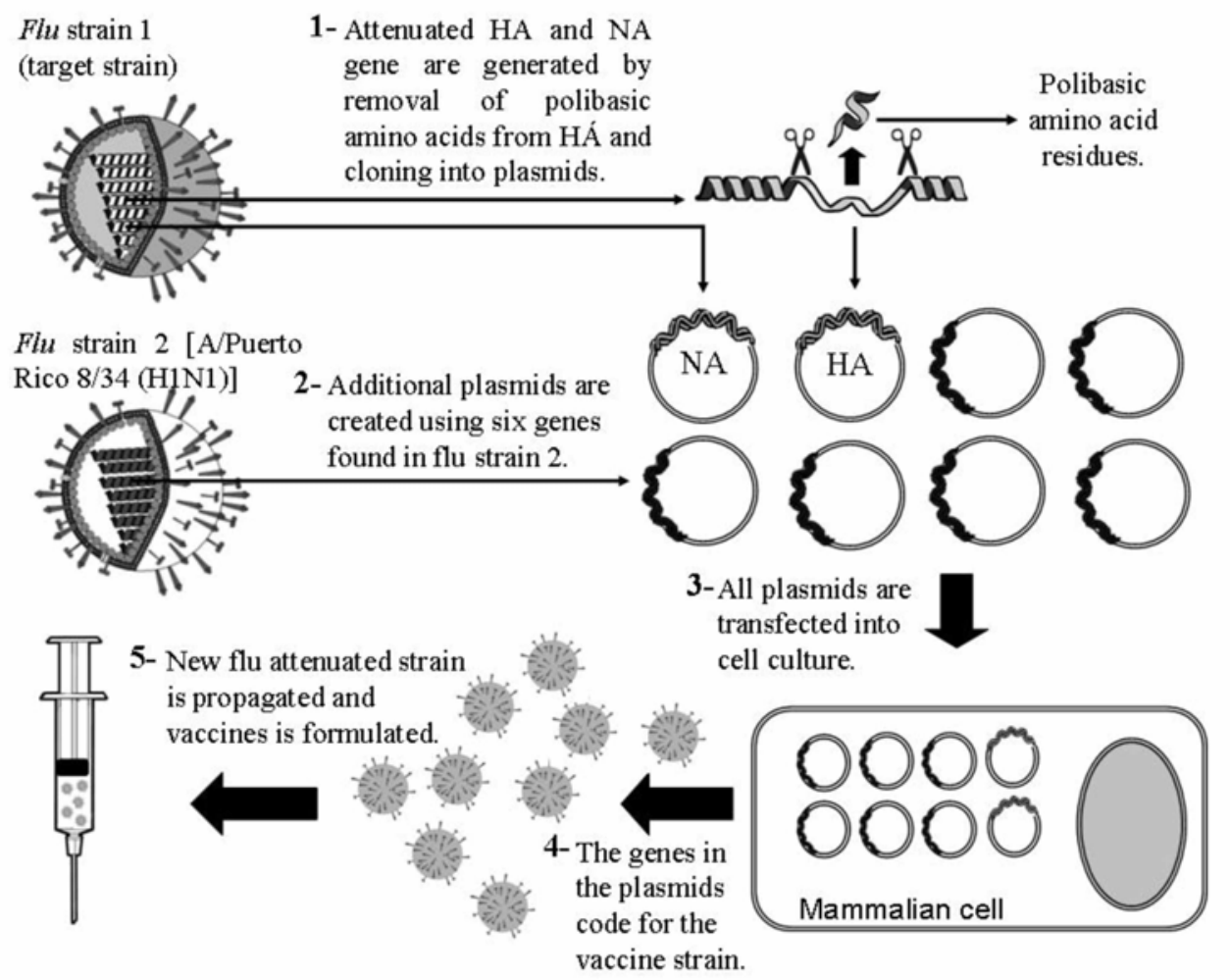

potential for clinical use in a pandemic situation caused by H5N1. Doses recommended for patients with confirmed or strongly suspected H5N1 infection are: $75 \mathrm{mg}$ oseltamivir (available as a capsule of $75 \mathrm{mg}$ for oral use) for adults or weightadjusted doses for children ( $30 \mathrm{mg}$ for $\leq 15 \mathrm{~kg}, 45 \mathrm{mg}$ for $>15$ to $23 \mathrm{~kg}, 60 \mathrm{mg}$ for $>23$ to $40 \mathrm{~kg}$ and $75 \mathrm{mg}$ for $>40 \mathrm{~kg}$ ); or $10 \mathrm{mg}$ of zanamivir (available only for oral inhalation by means of a diskhaler device) in adults and children $\geq 7$ years, twice daily for 5 days [83]. In high risks exposure groups, such as health care works, oseltamivir or zanamivir can be administered as chemoprophylaxis at the same doses, once daily for 7-10 days after the last known exposure. Of note, oseltamivir prophylaxis can safely be extended for up to 8 weeks [83].

Remarkably, emergence of resistance to these drugs is uncommon [74]. However, recent studies have described resistance to oseltamivir of H5N1 virus strains $[84,85]$. Oseltamivir-resistant H5N1 was found to have a tyrosine at position 274 in their neuraminidase [85]. Ferrets infected with this virus had lower virus titer in tissues when compared with ferrets infected with oseltamivir-sensitive H5N1 virus. Oseltamivir-resistant $\mathrm{H} 5 \mathrm{~N} 1$ virus was isolated from two of eight Vietnamese patients during oseltamivir treatment, and both patients died of influenza A infection, despite early initiation of treatment [84]. These results suggest that resistance to neuraminidase inhibitors can indeed emerge during a pandemic of H5N1, indicating that association of other antiviral agents should be considered for combination therapy of influenza A (H5N1) virus infections.
In the initial phases of an influenza pandemic, before a vaccine is made available, antiviral drugs will likely be essential to reduce spread [86]. The WHO has a stock of oseltamivir for deployment to the area of the initial outbreak. Nevertheless, stockpiling a neuraminidase inhibitor should be of concern to all countries in preparation for an influenza H5N1 pandemic.

\section{Concluding Remarks}

The H5N1 is an avian influenza virus that has infected wild birds and poultry in several places in the world, becoming a serious economic problem to many countries. This virus has caused cases of serious and frequently fatal illness in humans, but the transmission has been limited and virtually always has involved contact with infected birds. Several lines of evidence indicate that the threat of an $\mathrm{H} 5 \mathrm{~N} 1$ pandemic is real and that careful planning should be done in order for effective measures to be implemented to reduce the public health impact of H5N1. Monitoring and rapid detection of H5N1 is necessary to combat the spread of this virus. Recent human isolates resistant to adamantanes and neuraminidase inhibitors, as well as the difficulty for vaccine production against H5N1 illustrate the need for more research about the epidemiology and biology of H5N1 influenza virus.

\section{References}

1. Lamb R.A., Krug R.M., Orthomyxoviridae: The viruses and their replication. In: D.M. Knipe, et al. Editors Fields Virology. Philadelphia, PA, USA: Lippincott Williams \& Wilkins, pp. 1487-531, 2001. 
2. (WHO), W.H.O. Cumulative Number of Confirmed Human Cases of Avian Influenza A/(H5N1) Reported to WHO. 2006 [accessed April 10 2007].

3. Wright P.F., R.G. Webster, Orthomyxoviruses. In: D.E. Griffin, et al., Editors Fields Virology. Philadelphia, PA, USA: Lippincott Williams \& Wilkins, pp. 1533-79, 2001.

4. (ICTV). Orthomyxoviridae. In: ICTVdB - The Universal Virus Database, version 3. Büchen-Osmond, C. (Ed), ICTVdB Management, Columbia University, New York, USA 2004 [accessed September 25 2006].

5. Noda T., H. Sagara, A. Yen, et al. Architecture of ribonucleoprotein complexes in influenza A virus particles. Nature 2006;439(7075): 490-2.

6. Takeda M., G.P. Leser, C.J. Russell, et al. Influenza virus hemagglutinin concentrates in lipid raft microdomains for efficient viral fusion. Proc Natl Acad Sci U S A 2003;100(25):14610-7.

7. Chen, J., K.H. Lee, D.A. Steinhauer, et al., Structure of the hemagglutinin precursor cleavage site, a determinant of influenza pathogenicity and the origin of the labile conformation. Cell 1998;95(3):409-17.

8. Kido H., M. Murakami, K. Oba, et al. Cellular proteinases trigger the infectivity of the influenza A and Sendai viruses. Mol Cells 1999;9(3):235-44.

9. Gamblin S.J., L.F. Haire, R.J. Russell, et al. The structure and receptor binding properties of the 1918 influenza hemagglutinin. Science 2004;303(5665):1838-42.

10. Steinhauer D.A. Role of hemagglutinin cleavage for the pathogenicity of influenza virus. Virology 1999;258(1):1-20.

11. Li Z., Y. Jiang, P. Jiao, et al. The NS1 Gene Contributes to the Virulence of H5N1 Avian Influenza Viruses. J Virol 2006.

12. Salomon R., J. Franks, E.A. Govorkova, et al. The polymerase complex genes contribute to the high virulence of the human H5N1 influenza virus isolate A/Vietnam/1203/04. J Exp Med 2006;203(3):689-97.

13. Fouchier R.A., V. Munster, A. Wallensten, et al. Characterization of a novel influenza A virus hemagglutinin subtype (H16) obtained from black-headed gulls. J Virol, 2005;79(5):2814-22.

14. Lipatov A.S., E.A. Govorkova, R.J. Webby, et al. Influenza: emergence and control. J Virol, 2004;78(17):8951-9.

15. Arruda E., O.A.L. Cintra, F.G. Hayden. Respiratory Tract Viral Infections. In: R.L. Guerrant, D.H. Walker, and P.F. Weller, Editors Tropical infectious Diseases: Principles, Pathogens \& Practice. Philadelphia, PA, USA: Elsevier Churchill Livingstone, pp. 637-79, 2006.

16. Al Faress S., G. Cartet, O. Ferraris, et al. Divergent genetic evolution of hemagglutinin in influenza A H1N1 and A H1N2 subtypes isolated in the south-France since the winter of 2001-2002. J Clin Virol 2005;33(3):230-6.

17. Ghedin E., N.A. Sengamalay, M. Shumway, et al. Large-scale sequencing of human influenza reveals the dynamic nature of viral genome evolution. Nature, 2005;437(7062):11626.

18. Guan Y., M. Peiris, K.F. Kong, et al. H5N1 influenza viruses isolated from geese in Southeastern China: evidence for genetic reassortment and interspecies transmission to ducks. Virology 2002;292(1):16-23.

19. Li K.S., Y. Guan, J. Wang, et al. Genesis of a highly pathogenic and potentially pandemic H5N1 influenza virus in eastern Asia. Nature 2004;430(6996):209-13.

20. Hinshaw V.S., R.G. Webster, W.J. Bean, et al. The ecology of influenza viruses in ducks and analysis of influenza viruses with monoclonal antibodies. Comp Immunol Microbiol Infect Dis 1980;3(1-2):155-64.

21. Hinshaw V.S., R.G. Webster, B.C. Easterday, et al. Replication of avian influenza A viruses in mammals. Infect Immun 1981;34(2):354-61.
22. Nestorowicz A., Y. Kawaoka, W.J. Bean, et al. Molecular analysis of the hemagglutinin genes of Australian H7N7 influenza viruses: role of passerine birds in maintenance or transmission? Virology 1987; 160(2):411-8.

23. Webster R.G., V.S. Hinshaw, W.J. Bean, et al. Characterization of an influenza A virus from seals. Virology 1981;113(2):712-24.

24. Leneva I.A., O. Goloubeva, R.J. Fenton, et al. Efficacy of zanamivir against avian influenza A viruses that possess genes encoding H5N1 internal proteins and are pathogenic in mammals. Antimicrob Agents Chemother, 2001;45(4):1216-24.

25. Webster R.G., W.J. Bean, O.T. Gorman, et al. Evolution and ecology of influenza A viruses. Microbiol Rev 1992;56(1):152-79.

26. Reid A.H., T.G. Fanning, R.D. Slemons, et al. Relationship of pre1918 avian influenza HA and NP sequences to subsequent avian influenza strains. Avian Dis, 2003;47(3 Suppl):921-5.

27. Reid A.H., J.K. Taubenberger, T.G. Fanning, Evidence of an absence: the genetic origins of the 1918 pandemic influenza virus. Nat Rev Microbiol 2004;2(11):909-14.

28. Taubenberger J.K., A.H. Reid, R.M. Lourens, et al. Characterization of the 1918 influenza virus polymerase genes. Nature 2005;437(7060):889-93.

29. Taubenberger J.K., A.H. Reid, T.G. Fanning, Capturing a killer flu virus. Sci Am, 2005;292(1):48-57.

30. (WHO) W.H.O. H5N1 avian influenza: timeline. 2006 [accessed September 26 2006].

31. Kurtz J., R.J. Manvell, J. Banks, Avian influenza virus isolated from a woman with conjunctivitis. Lancet 1996;348(9031):901-2.

32. Webster R.G., J. Geraci, G. Petursson, et al. Conjunctivitis in human beings caused by influenza A virus of seals. N Engl J Med 1981;304(15):911.

33. Subbarao K., A. Klimov, J. Katz, et al. Characterization of an avian influenza A (H5N1) virus isolated from a child with a fatal respiratory illness. Science 1998;279(5349):393-6.

34. Matrosovich M., N. Zhou, Y. Kawaoka, et al. The surface glycoproteins of $\mathrm{H} 5$ influenza viruses isolated from humans, chickens, and wild aquatic birds have distinguishable properties. J Virol 1999;73(2):1146-55,

35. Maines T.R., X.H. Lu, S.M. Erb, et al. Avian influenza (H5N1) viruses isolated from humans in Asia in 2004 exhibit increased virulence in mammals. J Virol 2005;79(18):11788-800.

36. Butler D. Thai dogs carry bird-flu virus, but will they spread it? Nature 2006;439(7078):773.

37. Choi Y.K., T.D. Nguyen, H. Ozaki, et al. Studies of H5N1 influenza virus infection of pigs by using viruses isolated in Vietnam and Thailand in 2004. J Virol 2005;79(16):10821-5.

38. Ito T., J.N. Couceiro, S. Kelm, et al. Molecular basis for the generation in pigs of influenza A viruses with pandemic potential. J Virol 1998;72(9):7367-73

39. Rimmelzwaan G.F., D. van Riel, M. Baars, et al. Influenza A virus (H5N1) infection in cats causes systemic disease with potential novel routes of virus spread within and between hosts. Am J Pathol 2006;168(1):176-83.

40. Webster R.G., M. Peiris, H. Chen, et al. H5N1 outbreaks and enzootic influenza. Emerg Infect Dis 2006;12(1):3-8.

41. Webster R.G., M. Yakhno, V.S. Hinshaw, et al. Intestinal influenza: replication and characterization of influenza viruses in ducks. Virology 1978;84(2):268-78.

42. Guan Y., L.L. Poon, C.Y. Cheung, et al. H5N1 influenza: a protean pandemic threat. Proc Natl Acad Sci U S A 2004;101(21):815661.

43. Sturm-Ramirez K.M., T. Ellis, B. Bousfield, et al. Reemerging H5N1 influenza viruses in Hong Kong in 2002 are highly pathogenic to ducks. J Virol 2004;78(9):4892-901.

44. Peiris J.S., W.C. Yu, C.W. Leung, et al. Re-emergence of fatal human influenza A subtype $\mathrm{H} 5 \mathrm{~N} 1$ disease. Lancet 2004;363(9409):617-9.

45. Normile D., Avian influenza. Human transmission but no pandemic in Indonesia. Science 2006;312(5782):1855. 
46. Beigel J.H., J. Farrar, A.M. Han, et al. Avian influenza A (H5N1) infection in humans. N Engl J Med 2005;353(13):1374-85.

47. Mounts A.W., H. Kwong, H.S. Izurieta, et al. Case-control study of risk factors for avian influenza A (H5N1) disease, Hong Kong, 1997. J Infect Dis 1999;180(2):505-8.

48. (WHO), W.H.O. Epidemic and Pandemic Alert a Response. 2006 [accessed September 26 2006].

49. Tran T.H., T.L. Nguyen, T.D. Nguyen, et al. Avian influenza A (H5N1) in 10 patients in Vietnam. $\mathrm{N}$ Engl $\mathrm{J}$ Med 2004;350(12):1179-88.

50. Ungchusak K., P. Auewarakul, S.F. Dowell, et al. Probable personto-person transmission of avian influenza A (H5N1). N Engl J Med 2005;352(4):333-40.

51. Chan P.K., Outbreak of avian influenza A(H5N1) virus infection in Hong Kong in 1997. Clin Infect Dis 2002;34 Suppl 2:S58-64.

52. de Jong M.D., V.C. Bach, T.Q. Phan, et al. Fatal avian influenza A (H5N1) in a child presenting with diarrhea followed by coma. N Engl J Med 2005;352(7):686-91.

53. Stevens J., O. Blixt, T.M. Tumpey, et al. Structure and receptor specificity of the hemagglutinin from an H5N1 influenza virus. Science 2006;312(5772):404-10.

54. Hatta M., P. Gao, P. Halfmann, et al. Molecular basis for high virulence of Hong Kong H5N1 influenza A viruses. Science 2001;293(5536):1840-2.

55. Shinya K., S. Hamm, M. Hatta, et al. PB2 amino acid at position 627 affects replicative efficiency, but not cell tropism, of Hong Kong H5N1 influenza A viruses in mice. Virology 2004;320(2):258-66.

56. Guan Y., J.S. Peiris, A.S. Lipatov, et al. Emergence of multiple genotypes of H5N1 avian influenza viruses in Hong Kong SAR. Proc Natl Acad Sci U S A 2002;99(13):8950-5.

57. Yuen K.Y., P.K. Chan, M. Peiris, et al. Clinical features and rapid viral diagnosis of human disease associated with avian influenza A H5N1 virus. Lancet 1998;351(9101):467-71.

58. Lu P.S. Early diagnosis of avian influenza. Science 2006;312(5772):337.

59. Ng E.K., P.K. Cheng, A.Y. Ng, et al. Influenza A H5N1 detection. Emerg Infect Dis 2005;11(8):1303-5.

60. Cooper L.A. K. Subbarao. A simple restriction fragment length polymorphism-based strategy that can distinguish the internal genes of human H1N1, H3N2, and H5N1 influenza A viruses. J Clin Microbiol 2000;38(7):2579-83.

61. Russell R.J., L.F. Haire, D.J. Stevens, et al. The structure of H5N1 avian influenza neuraminidase suggests new opportunities for drug design. Nature 2006;443(7107):45-9.

62. Gao W., A.C. Soloff, X. Lu, et al. Protection of mice and poultry from lethal H5N1 avian influenza virus through adenovirusbased immunization. J Virol 2006;80(4):1959-64.

63. (WHO), W.H.O. WHO interim guideliness on clinical management of humans infected by influenza A (H5N1). 2004 [accessed april 03 2006].

64. Takada A., N. Kuboki, K. Okazaki, et al. Avirulent Avian influenza virus as a vaccine strain against a potential human pandemic. $\mathrm{J}$ Virol 1999;73(10):8303-7.

65. (CDC) C.f.D.C.a.P. Questions \& Answers: The Nasal-Spray Flu Vaccine (Live Attenuated Influenza Vaccine [LAIV]). 2003 [accessed april 3 2006].

66. Hoffmann E., A.S. Lipatov, R.J. Webby, et al. Role of specific hemagglutinin amino acids in the immunogenicity and protection of H5N1 influenza virus vaccines. Proc Natl Acad Sci U S A 2005;102(36):12915-20.

67. Horimoto T., A. Takada, K. Fujii, et al. The development and characterization of $\mathrm{H} 5$ influenza virus vaccines derived from a 2003 human isolate. Vaccine 2005.
68. Webby R.J., D.R. Perez, J.S. Coleman, et al. Responsiveness to a pandemic alert: use of reverse genetics for rapid development of influenza vaccines. Lancet 2004;363(9415):1099-103.

69. Neumann G., K. Fujii, Y. Kino, et al. An improved reverse genetics system for influenza A virus generation and its implications for vaccine production. Proc Natl Acad Sci U S A 2005;102(46):16825-9.

70. Epstein S.L., W.P. Kong, J.A. Misplon, et al. Protection against multiple influenza $A$ subtypes by vaccination with highly conserved nucleoprotein. Vaccine 2005;23(4647):5404-10.

71. Johansson B.E. Immunization with influenza A virus hemagglutinin and neuraminidase produced in recombinant baculovirus results in a balanced and broadened immune response superior to conventional vaccine. Vaccine 1999;17(15-16):2073-80.

72. Lambkin R., J.S. Oxford, S. Bossuyt, et al. Strong local and systemic protective immunity induced in the ferret model by an intranasal virosome-formulated influenza subunit vaccine. Vaccine 2004;22(31-32):4390-6.

73. De Filette M., W. Min Jou, A. Birkett, et al. Universal influenza A vaccine: optimization of M2-based constructs. Virology 2005;337(1):149-61.

74. Hayden F.G. P. Palese. Influenza virus. In: D.D. Richman, R.J. Whitley, and F.G. Hayden, Editors Clinical Virology. Washington, DC, USA: ASM Press, pp. 891-920, 2002.

75. Hayden F.G., A.J. Hay. Emergence and transmission of influenza A viruses resistant to amantadine and rimantadine. Curr Top Microbiol Immunol 1992;176:119-30.

76. Arruda E., F.G. Hayden. Update on therapy of influenza and rhinovirus infections. Adv Exp Med Biol 1996;394:175-87.

77. Bossart-Whitaker P., M. Carson, Y.S. Babu, et al. Threedimensional structure of influenza A N9 neuraminidase and its complex with the inhibitor 2-deoxy 2,3-dehydro-N-acetyl neuraminic acid. J Mol Biol 1993;232(4):1069-83.

78. Varghese J.N., J.L. McKimm-Breschkin, J.B. Caldwell, et al. The structure of the complex between influenza virus neuraminidase and sialic acid, the viral receptor. Proteins 1992;14(3):327-32.

79. von Itzstein M., W.Y. Wu, G.B. Kok, et al. Rational design of potent sialidase-based inhibitors of influenza virus replication. Nature 1993;363(6428):418-23.

80. Colman P.M., P.A. Hoyne, M.C. Lawrence. Sequence and structure alignment of paramyxovirus hemagglutinin-neuraminidase with influenza virus neuraminidase. J Virol 1993;67(6):2972-80.

81. Govorkova E.A., I.A. Leneva, O.G. Goloubeva, et al. Comparison of efficacies of RWJ-270201, zanamivir, and oseltamivir against H5N1, H9N2, and other avian influenza viruses. Antimicrob Agents Chemother 2001;45(10):2723-32.

82. Leneva, I.A., N. Roberts, E.A. Govorkova, et al. The neuraminidase inhibitor GS4104 (oseltamivir phosphate) is efficacious against A/Hong Kong/156/97 (H5N1) and A/Hong Kong/1074/99 (H9N2) influenza viruses. Antiviral Res 2000;48(2):101-15.

83. (WHO), W.H.O. WHO Rapid Advice Guidelines on pharmacological management of humans infected with avian influenza A (H5N1) virus 2006 [accessed October 13 2006].

84. de Jong M.D., T.T. Tran, H.K. Truong, et al. Oseltamivir resistance during treatment of influenza A (H5N1) infection. N Engl J Med 2005;353(25):2667-72.

85. Le Q.M., M. Kiso, K. Someya, et al. Avian flu: isolation of drugresistant H5N1 virus. Nature 2005;437(7062):1108.

86. Lee V.J., K.H. Phua, M.I. Chenm, et al. Economics of neuraminidase inhibitor stock piling for pandemic influenza, Singapore. Emerg Infect Dis 2006;12(1):95-102. 